\title{
Benchmarking workflows to assess performance and suitability of germline variant calling pipelines in clinical diagnostic assays.
}

Vandhana Krishnan ${ }^{1,2}$, ${ }^{+}$Sowmi Utiramerur ${ }^{3}$, Zena $\mathrm{Ng}^{3}$, Somalee Datta ${ }^{2}$, Michael P. Snyder ${ }^{1,2}$, Euan A. Ashley ${ }^{1,4,5}$

${ }^{1}$ Department of Genetics, School of Medicine, Stanford University, Stanford, CA

${ }^{2}$ Stanford Center for Genomics and Personalized Medicine, Stanford University, Palo Alto,

CA

${ }^{3}$ Clinical Genomics Program, Stanford Health Care, Stanford, CA

${ }^{4}$ Department of Cardiovascular Medicine

${ }^{5}$ Department of Biomedical Data Science

Current address of Somalee Datta: School of Medicine, Information Resources and Technology (IRT) - Research IT, Stanford University, Palo Alto, CA

Email address of Vandhana Krishnan: vandhana.krishnan@stanford.edu

Email address of Zena $\mathrm{Ng}$ : zng@stanfordhealthcare.org

Email address of Somalee Datta: somalee@stanford.edu

Email address of Michael P. Snyder: mpsnyder@stanford.edu

Email address of Euan A. Ashley: euan@stanford.edu

†Corresponding author: Sowmi Utiramerur: Email: sutiramerur@ stanfordhealthcare.org 


\section{Abstract}

Benchmarking the performance of complex analytical pipelines is an essential part of developing Laboratory Developed Assays (LDT). Reference samples and benchmark calls published by Genome in a Bottle (GIAB) Consortium have enabled the evaluation of analytical methods. However, the performance of such methods is not uniform across the different regions of the genome/exome and different variant types and lengths. Here we present a scalable and reproducible, cloud-based benchmarking workflow that can be used by clinical laboratories to rapidly access and validate the performance of LDT assays, across their regions of interest and reportable range, using a broad set of benchmarking samples.

\section{Keywords}

Benchmarking, workflow, GIAB reference genomes, precision, recall, truth set, docker, germline variants, laboratory developed assays

\section{Background}

Next Generation Sequencing (NGS) and analytical methods developed to detect various forms disease-causing polymorphisms are now routinely being used by clinical laboratories to determine the molecular etiology of complex diseases/disorders and in many cases to make critical treatment course decisions. In the past two decades, many polymorphisms in the human genome have been identified and validated that serve as predictive, diagnostic, and prognostic markers for complex inherited diseases. These genomic disease markers can be of different forms such as Single Nucleotide Variants (SNVs), small INsertions and DELetions (INDELs), large deletions and duplications (del/dups), and Copy Number Variations (CNVs) and can vary in size from a single base change to several Mega Bases (MB) in length and even whole chromosomal polysomy. Clinically relevant polymorphisms occur in different regions of the genome, including exonic, splice-sites, and deep-intronic regions. These polymorphisms also happen in various forms, including single base changes within high entropic regions, copy number changes to homopolymer repeats and copy number changes to Short Tandem Repeat (STR) regions. NGS platforms used to detect these polymorphisms; owing to their different sequencing chemistry and signal processing methods; have very different error modes and hence very different analytical performance across the different regions of the genome. Consequently, analytical methods specific to various NGS platforms such as Illumina, Ion Torrent, Pacific Biosciences, and Oxford Nanopore have been developed to both account for and correct the errors particular to these sequencing platforms. This has resulted in a dizzying array of combinations of sequencing platforms and analytical methods available to a clinical diagnostic laboratory to develop their LDT assay.

Benchmarking methods and pipelines are essential to accurately assess the performance of sequencing platforms and analytical methods before they are incorporated into clinical diagnostic assays. Genome In A Bottle (GIAB) consortium hosted by NIST has characterized the pilot genome (NA12878/HG001) (1) and six samples from the Personal Genome Project (PGP) (2). These benchmark calls for SNVs and small INDELs (1-20bp) from reference samples can be used for optimization, performance estimation, and analytical validation of LDT assays using complex analytical pipelines with multiple methods to detect polymorphisms in the genome. Global Alliance for Genomics and Health (GA4GH) benchmarking team have developed standardized tools (3) to evaluate the performance metrics of germline variant callers used primarily in research applications. 
Clinical Laboratory Improvement Amendments (CLIA) requires all laboratories using LDT to establish the test's performance specifications such as analytical sensitivity, specificity, reportable range, and reference range (4). College of American Pathologist (CAP) laboratory standards for NGS based clinical diagnostic (5) not only require the laboratories to assess and document the performance characteristics of all variants within the entire reportable range of LDTs but also obtain the performance characteristics for every type and size of variants that are reported by the assay. Laboratories are also required to assess the performance characteristics for clinically relevant variants such as $\Delta F 508$ and IVS8-5T (6) mutations in a CFTR assay. CAP guidelines also require laboratories to periodically (determined by the laboratory) assess and document the analytical performance characteristics to ensure that the LDT is continuing to perform as expected over time.

Benchmarking workflows/pipelines that are highly scalable, reproducible and capable of reporting the performance characteristics using a large number of reference and clinical samples within multiple highly stratified regions of interest are essential for clinical laboratories to optimize and routinely assess the performance of their LDT assays.

\section{Results}

Our goal was to develop a benchmarking workflow that any clinical laboratory could use to quickly evaluate and compare the performance characteristics of all suitable secondary analysis pipelines. Benchmarking workflow should further help optimize the analytical pipeline based on well-defined performance metrics and finally produce a thorough analytical validation report to justify the use of the analytical pipeline in their diagnostic assay to regulatory authorities such as CLIA and CAP.

To test the abilities of our benchmarking workflow, we used it to compare two analytical pipelines commonly used for germline variant calling 1. Pipeline based on Broad Institute's best practices guidelines using GATK HaplotypeCaller v3.7 and 2. SpeedSeq pipeline (7) based on FreeBayes v0.9.10 (8) as the primary variant calling engine. GATK HaplotypeCaller based pipeline was chosen over the FreeBayes pipeline as it out-performed in the detection of small-INDELs ( $1-20$ base pairs).

The performance characteristics of the analytical pipeline using GATK v3.7 was further optimized using benchmarking metrics generated using the five GIAB reference samples and four GeT-RM samples (see Methods) with known pathogenic variants. Also, it is critical for the clinical laboratories developing NGS based LDT assays to accurately determine the reportable range to avoid misdiagnosis leading to wrong treatment decisions. To this effect, we evaluated the performance metrics using the benchmarking workflow in three distinct genomic regions of interest (see Methods for details).

Although we have the benchmarking results for the region, including coding exons in all the RefSeq genes, we have omitted those findings in this section and focus on the clinically relevant regions.

Table 1: Benchmarking metrics for SNPs within coding exons of clinically relevant $\sim 7000$ genes (as specified in Methods). 


\begin{tabular}{|l|l|l|l|l|l|l|l|l|l|}
\hline $\begin{array}{l}\text { GIAB } \\
\text { genome / } \\
\text { NIST ID }\end{array}$ & $\begin{array}{l}\text { Number } \\
\text { of bases }\end{array}$ & $\begin{array}{l}\text { Truth } \\
\text { total }\end{array}$ & TP & FP & FN & TN & NPA & Precision & Recall \\
\hline NA12878 & 13728555 & 7803 & 7781 & 4 & 22 & 13720748 & 100 & 99.95 & 99.72 \\
\hline NA24143 & 12549224 & 7470 & 7460 & 14 & 10 & 12541740 & 100 & 99.81 & 99.87 \\
\hline NA24149 & 12538042 & 7495 & 7485 & 19 & 9 & 12530529 & 100 & 99.75 & 99.88 \\
\hline NA24385 & 12626866 & 7452 & 7436 & 0 & 16 & 12619414 & 100 & 100 & 99.79 \\
\hline NA24631 & 12808688 & 7591 & 7581 & 6 & 10 & 12801091 & 100 & 99.92 & 99.87 \\
\hline
\end{tabular}

102
Table 2: Benchmarking metrics for SNPs in whole exome regions, including non-coding exons, splice sites (+/- 20 bp) and clinically relevant deep intronic regions.

\begin{tabular}{|l|l|l|l|l|l|l|l|l|l|}
\hline $\begin{array}{l}\text { GIAB } \\
\text { genome / } \\
\text { NIST ID }\end{array}$ & $\begin{array}{l}\text { Number } \\
\text { of bases }\end{array}$ & $\begin{array}{l}\text { Truth } \\
\text { total }\end{array}$ & TP & FP & FN & TN & NPA & Precision & Recall \\
\hline NA12878 & 71152019 & 57822 & 57024 & 491 & 776 & 71093728 & 100 & 99.15 & 98.66 \\
\hline NA24143 & 65657646 & 55975 & 55340 & 669 & 611 & 65601026 & 100 & 98.81 & 98.91 \\
\hline NA24149 & 65597266 & 55518 & 54827 & 669 & 669 & 65541101 & 100 & 98.79 & 98.79 \\
\hline NA24385 & 65948744 & 56068 & 55329 & 389 & 705 & 65892321 & 100 & 99.30 & 98.74 \\
\hline NA24631 & 66988987 & 56948 & 56303 & 394 & 643 & 66931647 & 100 & 99.31 & 98.87 \\
\hline
\end{tabular}

Tables 1 and 2 show the benchmarking metrics for SNPs in all five GIAB samples within the clinically relevant genes and whole exome regions, respectively. The precision, recall, and NPA metrics for SNPs are uniform across all the reference samples, and there is no sample bias in the results for some of the better-characterized samples such as NA24385 and NA12878. Performance metrics for SNPs within the clinically relevant gene region is significantly better than those within the whole exome region. Recall metrics, in particular, are a percentage point better in the clinically pertinent gene region, across all reference samples. This is attributable to the fact that many genes have isoforms, resulting in higher alignment errors, and some genes have either very high or very low GC content, resulting in higher than average sequencing errors within these regions of the genome. The finding is of great clinical significance, since the reportable region of most inherited disease/disorder, LDT assay is limited to the clinically relevant genes and thereby the overall performance characteristics of the assay is better than that estimated over either the whole genome or whole exome regions.

Table 3: Benchmarking metrics for indels of different size ranges in NA24385 (truth set NIST v3.3.2, total bases $=12,626,866$ ) for the regions within $\sim 7000$ clinically relevant genes (as specified in Methods).

\begin{tabular}{|l|l|l|l|l|l|l|l|l|}
\hline $\begin{array}{l}\text { Size of indels } \\
\text { in NA24385 }\end{array}$ & $\begin{array}{l}\text { Truth } \\
\text { total }\end{array}$ & TP & FP & FN & TN & NPA & Precision & Recall \\
\hline $1-10$ & 145 & 136 & 12 & 9 & 12626709 & 100 & 91.89 & 93.79 \\
\hline $11-20$ & 9 & 9 & 0 & 0 & 12626857 & 100 & 100 & 100 \\
\hline $21-50$ & 3 & 3 & 0 & 0 & 12626863 & 100 & 100 & 100 \\
\hline All Indels & 157 & 148 & 12 & 9 & 12626697 & 100 & 92.50 & 94.27 \\
\hline
\end{tabular}

Table 4: Benchmarking metrics on the number of indels of different size ranges in NA24385 (truth set NIST v3.3, total bases $=65,948,744$ ) for the whole exome regions including noncoding exons, splice sites (+/- 20 bp) and clinically relevant deep intronic regions. 


\begin{tabular}{|l|l|l|l|l|l|l|l|l|}
\hline $\begin{array}{l}\text { Size of indels } \\
\text { in NA24385 }\end{array}$ & $\begin{array}{l}\text { Truth } \\
\text { total }\end{array}$ & TP & FP & FN & TN & NPA & Precision & Recall \\
\hline $1-10$ & 5169 & 4727 & 872 & 442 & 65942703 & 100 & 84.43 & 91.45 \\
\hline $11-20$ & 203 & 188 & 10 & 15 & 65948531 & 100 & 94.95 & 92.61 \\
\hline $21-50$ & 67 & 56 & 3 & 11 & 65948674 & 100 & 94.92 & 83.58 \\
\hline All Indels & 5362 & 4920 & 885 & 468 & 65942471 & 100 & 84.75 & 91.27 \\
\hline
\end{tabular}

Tables 3 and 4 provide the indel benchmarking metrics for sample NA24385 in the clinically relevant and whole exome regions, respectively. As expected, the benchmarking workflow reveals that the performance metrics for INDELs are lower than those for SNPs. However, the stratification by INDEL size, helped us determine the reference range for INDELs (1- 20 base-pairs). The recall metric for INDELs larger than 20 base-pairs is significantly lower than the recall for INDELs 1 - 20 base-pairs. As in the case of SNPs, performance metrics for INDEL detection within the clinically relevant genes of interest is better than the whole exome region.

The benchmarking results of the other GIAB reference samples in the clinically relevant and whole exome regions can be obtained in the Supplementary Materials Table S1-S4 and Table S5-S8, respectively. The histogram for the indel size distribution in the NA24385 reference sample for the whole exome region is in Supplementary Material as Fig S1. The histograms of indel size distributions for GIAB samples in both the whole exome and clinically relevant regions are available in our github repository - vandhana/stanford-benchmarking-workflows.

Table 5: Validation of the presence of the truth variants in the GeT-RM samples (as specified in Methods) using our variant calling pipeline.

\begin{tabular}{|l|l|l|l|}
\hline $\begin{array}{l}\text { GeT-RM } \\
\text { Sample ID }\end{array}$ & Chromosome:Position & Truth Variant & $\begin{array}{l}\text { Truth Variant } \\
\text { Detected }\end{array}$ \\
\hline \multirow{3}{*}{ NA04408 } & $15: 91310152$ & TATC -> T & Yes \\
\cline { 2 - 4 } & $15: 91310156$ & T -> TA & Yes \\
\cline { 2 - 4 } & $15: 91310158$ & A -> ATTC & Yes \\
\hline NA14090 & $17: 41276044$ & ACT -> A & Yes \\
\hline NA14170 & $13: 32914437$ & GT -> G & Yes \\
\hline NA16658 & $10: 43609103$ & G -> T & Yes \\
\hline
\end{tabular}

Finally, our benchmarking workflow was able to confirm that our variant calling pipeline can detect all the clinical variants in GeT-RM samples listed in Table 5.

To get all the metrics produced by hap.py and other output files including plots from our benchmarking workflow for each reference sample, please refer to the Supplementary Data files.

\section{Discussion}

GIAB consortium has helped developed standards for genomic data to evaluate the performance of NGS sequencing platforms and analytical methods used for alignment and variant calling. The precisionFDA platform has enabled the genomics community to develop and deploy benchmarking tools that can evaluate the performance of analytical methods against the gold standard datasets. These benchmarking tools, along with accuracy challenges, has led to the development of highly accurate variant calling methods. However, the requirements of a clinical diagnostic laboratory go beyond the simple evaluation of 
163 performance characteristics of an analytical pipeline against one or more reference samples.

164 Our purpose was to build a benchmarking workflow to meet the assay optimization and validation needs of a clinical laboratory. The primary benefit of our benchmarking workflow is that it allows for the assay performance to be evaluated using a broad set of both reference samples with a large number of gold-standard variant calls and clinical samples with a small number of clinical variants, that are specific to the diagnostic assay being evaluated. The benchmarking workflows enable the clinical laboratories to establish the reporting range of the diagnostic assay by estimating the performance within multiple regions of interest.

Unlike web-based benchmarking apps, such as those provided by the precision FDA platform or GA4GH, our benchmarking framework can be seamlessly integrated with any variant calling pipeline in the user's software environment. Thus, our benchmarking workflows enable ease of use and avoid the transfer of sensitive data to different locations, which could be non-Protected Health Information (PHI) compliant.

Our benchmarking modules if integrated with deployment tools, such as Jenkins (9) and CircleCI (10), that work on the principle of continuous integration and continuous delivery/deployment (CI/CD), it provides a foolproof way of examining consistency in results. In this era where workflows generating reproducible results are gaining attention, easy incorporation of workflows with $\mathrm{CI} / \mathrm{CD}$ tools is a nice feature to have.

The benchmarking workflow is distributed using human-readable YAML (11) format, and it might limit direct porting to existing WDL based workflows such as those published by the Broad Institute $(12,13)$. Similarly, conversion of the benchmarking YAML files to Common Workflow Language (CWL) format is required to run workflows published by GA4GH (1416). However, since we have used docker images for the software tools used within the benchmarking framework, portability to other runtime environments should not take a significant effort for a bioinformatician.

\section{Conclusions}

Benchmarking variants is a critical part of implementing variant calling pipelines for research or clinical purposes. Here, we have successfully implemented benchmarking workflows that generate metrics such as specificity, precision, sensitivity for germline SNPs, and indels in whole exome sequencing data. Also, indel size distributions even in the form of histograms are provided. Combining these benchmarking results with validation using known variants of clinical significance in publicly available cell lines, we were able to establish our variant calling pipelines in a clinical setting. Our benchmarking workflow can serve as a plug-in to any existing variant calling pipeline to work as an integrated unit or be used as a separate module as well.

\section{Methods}


The benchmarking workflow, as illustrated in Figure 1, is a sequence of steps required to perform a rapid and comprehensive analytical validation of a clinical diagnostic assay based on germline variants. The benchmarking workflow can be easily integrated with any secondary-analysis pipeline used in a diagnostic assay to call germline variants, and our workflow accepts germline variants (SNVs and small INDELs) in Variant Call Format VCF v4.1(17) or higher. The workflow takes one or more stratification files specifying the regions of interest in BED (18) format and generates a comprehensive analytical validation report detailing the performance characteristics of the assay within each of the specified regions of interest. Benchmark variant calls that are considered as ground truths for each of the reference sample used to evaluate the analytical performance can be also be specified in VCF format.

\section{Figure 1. Schematic diagram of the benchmarking framework used in this study}

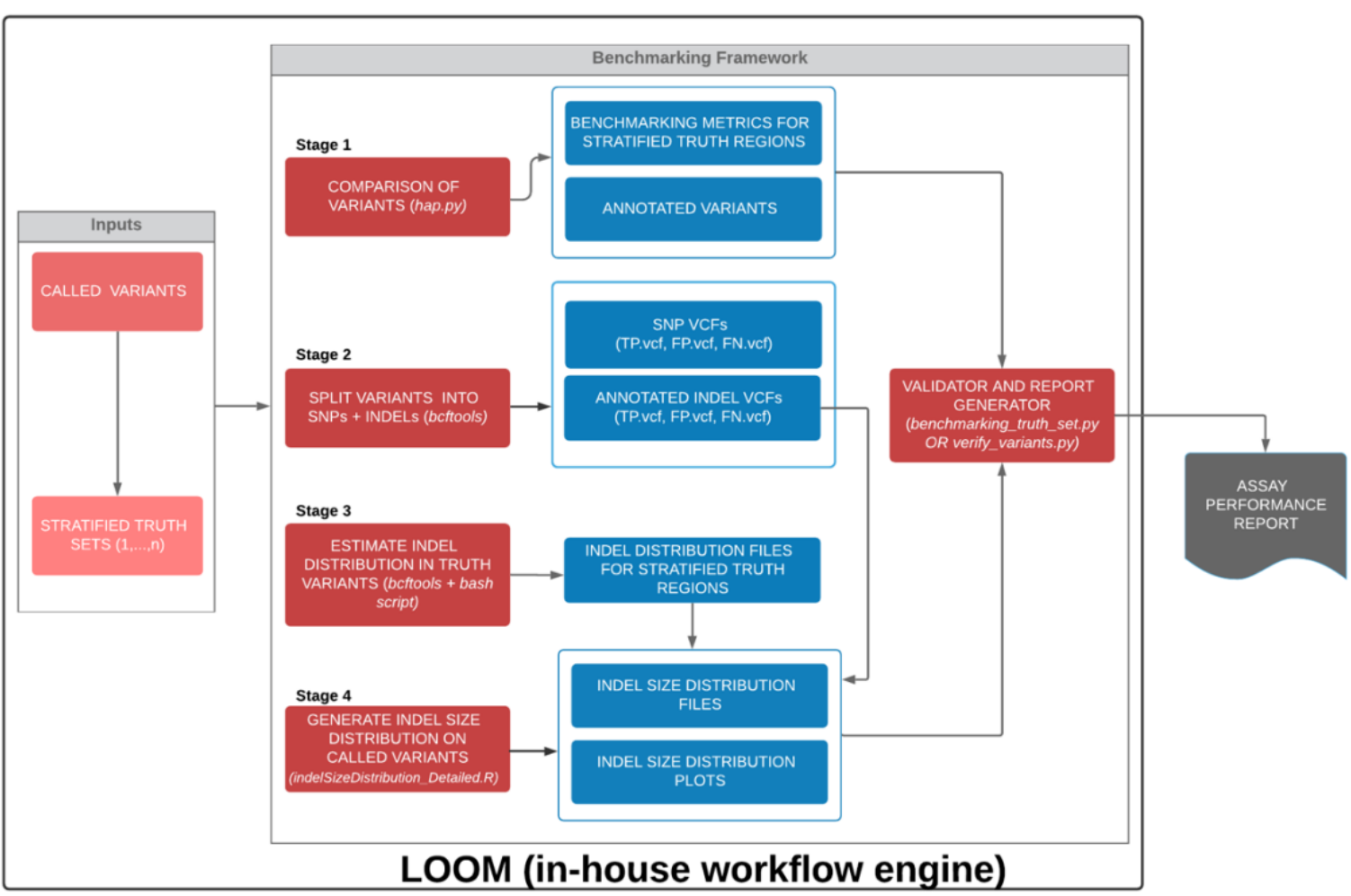

Figure 1 legend: All the stages in the benchmarking workflow have been dockerized. The docker images are available in DockerHub as specified in the Methods section.

The first step in the benchmarking process involves the comparison of input variants generated by the analytical pipeline with the benchmark variant calls within each region of interest. The variant calls are compared using hap.py $(19,20)$, which is capable of haplotype construction from individual genotype calls and is recommended by GIAB consortium and GA4GH. The variant comparison step is performed for each of the stratification or region of interest file specified as input, and hap.py generates a single output VCF file classifying the variant calls defined in the input and truth VCF files as either True Positive (TP), False Positive (FP) or False Negative (FN). 
Step two in the benchmarking workflow splits the variant calls annotated using hap.py by variant type (SNPs and small INDELs) and by variant classification (TP/FP/FN). This step is executed within the workflow for each of the stratification or region of interest file specified. The VCF files are split by variant type using bcftools (21), and a bash script is used to further split the variant calls by the variant classification. This allows the workflow to generate the performance metrics for each of the variant types reported by the diagnostic assay.

Steps two and three of the benchmarking workflow (see Figure 1.) were used to generate a histogram of small INDELs by size. The bins used for INDEL size histograms were a. 1 base-pair, b. 2-5 base-pairs, c. 6-10 base-pairs, d. 11 - 20 base-pairs, e. $21-50$ base-pairs, and f. Greater than 50 base-pairs. The R script - indelSizeDistribution_Detailed.R (code in Additional File 1) then calculates the performance metrics of the assay for each of the INDEL size bins. The Python script - benchmarking_truth_set.py (Additional File 2) consolidates the benchmarking metrics previously obtained, calculates the NPA related metrics combining some of the bin size ranges (user preferred) for all reference samples provided.

In addition to benchmarking call sets for well-characterized reference samples published by the GIAB consortium, the benchmarking workflow allows for clinical laboratories to specify addition samples with clinically relevant variants as ground truths to estimate the analytical performance of the assay for specific variant types such as $\Delta F 508$ and IVS8-5T in CFTR panels. Python script - verify_variants.py (Additional File 3) accepts the ground-truth variant call sets to confirm the presence/absence of these variants in the VCF files generated by the variant calling pipeline. The details on the usage of the above scripts and associated README file are available in our public repository (also see Supplementary Materials).

Finally, the benchmarking workflow generates a comprehensive analytical validation report using all the provide benchmarking ground-truth call sets.

\section{Scalability and Reproducibility of Benchmarking workflow}

The benchmarking workflow is designed to be repeatable and reproducible by using Docker containers for all software and bioinformatics components used within the workflow (see Table 6.). The workflow is distributed in human-readable data serialization format YAML v1.2, and the workflow can be readily executed using the workflow execution manager LOOM (22). The workflow definition file - Benchmarking.yaml (see Supplementary Materials) can also be easily ported to Common Workflow Language (CWL) or Workflow Definition Language (WDL) formats and can be executed using workflow execution managers such as Toil $(23,24)$ and Cromwell $(25)$.

Table 6. Docker containers and DockerHub repository location for each of the individual software components used in the benchmarking workflow.

\begin{tabular}{|l|l|}
\hline Software Component & Docker Container \\
\hline hap.py v 0.2.10 & sowmiu/happy:latest \\
\hline bcftools & vandhanak/bcftools:1.3.1 \\
\hline indelSizeDistribution_Detailed.R & vandhanak/rbase:3.3.2 \\
\hline
\end{tabular}




\section{Golden/ground-truth callsets}

The golden/ground-truth sets for five reference and PGP genomes are currently available NA12878 (CEPH family's daughter), NA24143 (AJ mother), NA24149 (AJ father), NA24385 (AJ son), and NA24631(Chinese son) and these reference call sets were used in this benchmarking study. GIAB provides a high confidence regions file and a high confidence VCF file, and as recommended by GIAB, only the high confidence calls were used in the evaluation of the assay's performance characteristics. The NIST versions and their corresponding FTP site locations used for the above samples in this study can be found in the Supplementary Material.

In addition to the GIAB reference samples, samples with known pathogenic germline variants (see Table 2.) for various inherited diseases/disorders were chosen from Genetic Testing Reference Materials Coordination Program (GeT-RM) (26-30)

Table 7. GeT-RM sample ids and location of ground-truth variants in GRCh37 coordinates.

\begin{tabular}{|l|l|l|}
\hline $\begin{array}{l}\text { GeT-RM Sample } \\
\text { ID }\end{array}$ & Chromosome:Position & $\begin{array}{l}\text { Truth } \\
\text { Variant }\end{array}$ \\
\hline \multirow{3}{*}{ NA04408 } & $15: 91310152$ & TATC -> T \\
\cline { 2 - 3 } & $15: 91310156$ & T -> TA \\
\cline { 2 - 3 } & $15: 91310158$ & A - ATC ATC \\
\hline NA14090 & $17: 41276044$ & ACT -> A \\
\hline NA14170 & $13: 32914437$ & GT -> G \\
\hline NA16658 & $10: 43609103$ & G -> T \\
\hline
\end{tabular}

\section{Benchmarking metrics}

\section{Stratification or Regions of Interest (ROI) BED files.}

Three stratification files were used to evaluate the performance characteristics of an inherited Whole Exome Sequencing (WES) assay.

1. Coding Exons for all known transcripts in RefSeq genes: RefSeq gene names, transcripts, and coordinates of all coding exons were obtained from the UCSC genome browser(31, 32).

2. Clinically relevant regions of the human genome: Clinically relevant regions were determined by intersecting coordinates of all known pathogenic variants reported in OMIM (33), ClinVar (34) and DECIPHER v9.28 (35) with the all exon regions (Coding and Non-Coding) file for RefSeq genes obtained from UCSC genome browser. The exonic coordinates were later extended by 20 base-pairs on either end to include canonical and non-canonical splice sites. Deep-intronic regions with pathogenic variants were added to the exonic regions to generate the final clinically relevant regions (BED) file.

3. Whole Exome regions file for RefSeq genes was obtained from UCSC genome browser. The exon regions were extended by 20 base-pairs on either end to include splice sites.

Precision and recall are benchmarking metrics provided as output by hap.py. The true positives (TP), false positives (FP), and false negatives (FN) are counted as described by the 
developers of hap.py (20). Again, as explained by the authors of hap.py, precision and recall are calculated using the below formulae:

Precision $=$ True Positives $/($ True Positives + False Positives $)$

Recall = True Positives/(True Positives + False Negatives $)$

Other metrics reported by hap.py such as variants outside the high confidence truth set regions and transition or transversion SNP type can be found in the extended.csv files included in the Supplementary Materials.

The total number of bases per sample in a particular region of interest as specified by the corresponding bed file was computed using a bash command provided in the Supplementary Materials.

True negatives (TN) and Total Negatives are computed using the following:

$T N=$ Total number of bases in the region of interest - (True Positives + False Positives + False Negatives)

Total Negatives $=$ True Negatives + False Positives

The Negative Percentage Agreement (NPA) or specificity as recommended by the FDA (36) is calculated using

NPA = True Negatives/Total Negatives

\section{List of abbreviations}

NIST - National Institute of Standards and Technology

GIAB - Genome in a bottle consortium

SNPs - Single nucleotide polymorphisms

Indels - insertions/deletions

WES - Whole Exome Sequencing

NPA - Negative Percent Agreement

TN - True Negative

TP - True Positive

FN - False Negative

FP - False Positive

OMIM - public database containing the human genes, their genetic phenotypes and associations with genetic disorders (Online Mendelian Inheritance in Man)

DECIPHER - public database with genotypic and phenotypic data from 30,000 individuals ClinVar - public database with information on the relationship between medically important variants and phenotypes.

\section{Declarations}


Ethics approval and consent to participate

377 Not applicable

\section{Consent for publication}

380 The authors declare that they have no competing interests.

\section{Availability of data and material}

The datasets generated and/or analyzed during the current study are available in the GitHub repository - vandhanak/stanford-benchmarking-workflows.

\section{Competing interests}

Not applicable

\section{Funding}

390 This work was funded by Stanford HealthCare, Stanford Children's Health and Stanford School of Medicine.

\section{Authors' contributions}

VK designed and implemented the benchmarking workflow. SU and VK wrote the manuscript. ZN implemented the scripts to generate the performance assay report including the clinical variant validation. SU, SD, MP and EA conceived, designed and supervised the overall study. All authors read and approved the final manuscript.

400 We thank Amin Zia for providing useful information during the initial phase of the

401 benchmarking work. We thank Nathan Hammond and Issac Liao for the development of the 402 in-house workflow engine "Loom" which was used to run the variant calling pipelines and

403 the subsequent benchmarking workflows.

404 We are thankful to Chittaranjan Muthumalai for leading the automated benchmarking pipeline testing efforts and Jason Merker for useful discussions in terms of clinical relevance during the benchmarking process.

This study makes use of data generated by the DECIPHER community. A full list of centers who contributed to the generation of the data is available from http://decipher.sanger.ac.uk and via email from decipher@sanger.ac.uk. Funding for the project was provided by the Wellcome Trust.

\section{References}

416 1. Zook JM, Chapman B, Wang J, Mittelman D, Hofmann O, Hide W, et al. Integrating 417 human sequence data sets provides a resource of benchmark SNP and indel genotype calls.

418 Nat Biotechnol. 2014;32(3):246-51.

419 2. Zook JM, Catoe D, McDaniel J, Vang L, Spies N, Sidow A, et al. Extensive 420 sequencing of seven human genomes to characterize benchmark reference materials. Sci 421 Data. 2016;3:160025. 
3. Krusche P, Trigg L, Boutros PC, Mason CE, De La Vega FM, Moore BL, et al. Best practices for benchmarking germline small-variant calls in human genomes. Nat Biotechnol. 2019;37(5):555-60.

4. Jennings L, Van Deerlin VM, Gulley ML, Committee CoAPMPR. Recommended principles and practices for validating clinical molecular pathology tests. Arch Pathol Lab Med. 2009;133(5):743-55.

5. N A, Q Z, L B, DK D, B F, JS G, et al. College of American Pathologists laboratory standards for next-generation sequencing clinical tests. Arch Pathol Lab Med. 2015;139(4):481-93.

6. MS W, GR C, RJ D, DA D, K K, M M, et al. Cystic fibrosis population carrier screening: 2004 revision of the American College of Medical Genetics mutation panel. Genetics in Medicine. 2004;6(5):387-91.

7. Chiang C, Layer RM, Faust GG, Lindberg MR, Rose DB, Garrison EP, et al. SpeedSeq: ultra-fast personal genome analysis and interpretation. Nat Methods. 2015;12(10):966-8.

8. Garrison E, Marth G. Haplotype-based variant detection from short-read sequencing. arXiv:1207.3907v2 [q-bio.GN]; 20 Jul 2012.

9. Jenkins; Available from: https://jenkins.io/doc/.

10. CircleCI; Available from: https://circleci.com/docs/.

11. YAML; Available from: https://yaml.org/Available from:

https://www.tutorialspoint.com/yaml/index.htm.

12. OpenWDL: Broad Institute; [Available from: https://software.broadinstitute.org/wdl/.

13. GATK workflows: Broad Institute; [Available from: https://github.com/gatkworkflows/.

14. Amstutz P, Crusoe MR, Tijanić N, Chapman B, Chilton J, Heuer M, et al. Common Workflow Language, v1.0. Specification, Common Workflow Language working group. In: Peter Amstutz MRC, Nebojša Tijanić, editor. 2016.

15. Common Workflow Language (CWL). Software Freedom Conservancy, Inc.

16. O'Connor BD, Yuen D, Chung V, Duncan AG, Liu XK, Patricia J, et al. The

Dockstore: enabling modular, community-focused sharing of Docker-based genomics tools and workflows. F1000Res. 2017;6:52.

17. Variant Call Format; Available from:

http://www.internationalgenome.org/wiki/Analysis/variant-call-format.

18. BED format; Available from: http://genome.ucsc.edu/FAQ/FAQformat\#format1.

19. Krusche P, Trigg L, Boutros PC, Mason CE, De La Vega FM, Moore BL, et al.

Author Correction: Best practices for benchmarking germline small-variant calls in human genomes. Nat Biotechnol. 2019;37(5):567.

20. Krusche P. Haplotype VCF Comparison Tools; Available from:

https://github.com/Illumina/hap.pyAvailable from:

https://github.com/Illumina/hap.py/blob/master/doc/happy.md.

21. BCFtools; Available from: http://samtools.github.io/bcftools/.

22. Hammond N. Loom: platform-independent tool to create, execute, track, and share workflows. 2017.

23. Vivian J. Toil; Available from: https://toil.readthedocs.io/en/latest/.

24. Vivian J, Rao AA, Nothaft FA, Ketchum C, Armstrong J, Novak A, et al. Toil enables reproducible, open source, big biomedical data analyses. Nat Biotechnol. 2017;35(4):314-6.

25. Cromwell: Broad Institute; Available from:

https://cromwell.readthedocs.io/en/stable/.

26. CDC. GeT-RM Home: Available from: https://wwwn.cdc.gov/clia/Resources/GETRM/default.aspx. 
27. GeT-RM NA04408: Coriell Institute of Medical Research; Available from: https://www.coriell.org/0/Sections/Search/Sample_Detail.aspx?Ref=NA04408\&Product=DN A. https://www.coriell.org/0/Sections/Search/Sample Detail.aspx?Ref=NA14090\&Product=DN A. 29.

Get-RM NA14170: Coriell Institute of Medical Research; Available from: https://www.coriell.org/0/Sections/Search/Sample_Detail.aspx?Ref=NA14170\&Product=DN A.

30. GeT-RM NA16658: Coriell Institute of Medical Research; Available from: https://www.coriell.org/0/Sections/Search/Sample_Detail.aspx?Ref=NA16658\&Product=DN

483 A. 31. Fujita PA, Rhead B, Zweig AS, Hinrichs AS, Karolchik D, Cline MS, et al. The UCSC Genome Browser database: update 2011. Nucleic Acids Res. 2011;39(Database issue):D876-82. 32. Karolchik D, Hinrichs AS, Kent WJ. The UCSC Genome Browser. Curr Protoc Bioinformatics. 2009; Chapter 1:Unit1.4.

489 33. Online Mendelian Inheritance in Man, OMIM ${ }^{\circledR} .2017$ ed: McKusick-Nathans Institute 490 of Genetic Medicine, Johns Hopkins University (Baltimore, MD).

491 34. Landrum MJ, Lee JM, Benson M, Brown G, Chao C, Chitipiralla S, et al. ClinVar: 492 public archive of interpretations of clinically relevant variants. Nucleic Acids Res.

493 2016;44(D1):D862-8.

494 35. Firth HV, Richards SM, Bevan AP, Clayton S, Corpas M, Rajan D, et al. DECIPHER: 495 Database of Chromosomal Imbalance and Phenotype in Humans Using Ensembl Resources.

496 Am J Hum Genet. 2009;84(4):524-33.

497 36. Administration USFaD. Guidance for industry and FDA staff: statistical guidance on reporting results from studies evaluating diagnostic tests. .

\section{Supplementary Materials}

\section{Preparation of truth sets for exome regions}

504 The NIST version and the ftp site used to download the original data for each of the GIAB samples (before preprocessing) used in this study are listed here.

NA12878

NIST v3.3: ftp://ftptrace.ncbi.nlm.nih.gov/giab/ftp/release/NA12878_HG001/NISTv3.3/NA12878_GIAB_highc onf_CG-IllFB-IllGATKHC-Ion-Solid-10X_CHROM1-X_v3.3_highconf.bed $\mathrm{ftp}: / / \mathrm{ftp}-$ trace.ncbi.nlm.nih.gov/giab/ftp/release/NA12878_HG001/NISTv3.3/NA12878_GIAB_highc onf_CG-IllFB-IllGATKHC-Ion-Solid-10X_CHROM1-X_v3.3_highconf.vcf.gz

\section{NA24143}

NIST v3.3: 
3/HG004_GIAB_highconf_CG-IllFB-IllGATKHC-Ion-10X_CHROM1-

NA24149

NIST v3.3:

ftp://ftp-

530 trace.ncbi.nlm.nih.gov/giab/ftp/release/AshkenazimTrio/HG003_NA24149_father/NISTv3.3/

531 HG003_GIAB_highconf_CG-IllFB-IllGATKHC-Ion-10X_CHROM1-22_v3.3_highconf.bed

532 ftp://ftp-

533 trace.ncbi.nlm.nih.gov/giab/ftp/release/AshkenazimTrio/HG003_NA24149_father/NISTv3.3/

534

535

536

537

HG003_GIAB_highconf_CG-IllFB-IllGATKHC-Ion-10X_CHROM1-

22 v3.3 highconf.vcf.gz

538 NIST v3.3:

539 ftp://ftp-

540 trace.ncbi.nlm.nih.gov/giab/ftp/release/AshkenazimTrio/HG002_NA24385_son/NISTv3.3/H

541 G002_GIAB_highconf_CG-IIlFB-IIlGATKHC-Ion-Solid-10X_CHROM1-

542 22_v3.3_highconf.bed

543 ftp://ftp-

544 trace.ncbi.nlm.nih.gov/giab/ftp/release/AshkenazimTrio/HG002_NA24385_son/NISTv3.3/H

545 G002_GIAB_highconf_CG-IIlFB-IIlGATKHC-Ion-Solid-10X_CHROM1-

$546 \quad 22$ v3.3 highconf.vcf.gz

NA24631

549 NIST v3.3.2:

550 ftp://ftp-

551 trace.ncbi.nlm.nih.gov/giab/ftp/release/ChineseTrio/HG005_NA24631_son/NISTv3.3.2/GRC h37/HG005_GRCh37_highconf_CG-IllFB-IllGATKHC-Ion-SOLID_CHROM122_v.3.3.2_highconf_noMetaSV.bed ftp://ftptrace.ncbi.nlm.nih.gov/giab/ftp/release/ChineseTrio/HG005_NA24631_son/NISTv3.3.2/GRC h37/HG005_GRCh37_highconf_CG-IllFB-IllGATKHC-Ion-SOLID_CHROM122_v.3.3.2_highconf.vcf.gz

\section{Bash command to compute total number of bases in a region of interest}

In the above command, <Consolidated.bed $>$ refers to GIAB original high confidence bed file for a sample intersected with the bed file of the region of interest such as coding exons, whole exome or clinically relevant gene regions. The user can use this command to calculate bases with their desired stratified region in the bed format which is required to compute metrics such as true negatives.

\section{Output files generated by Benchmarking workflow}


569 Our benchmarking workflow generates the following output files:

570 1. <Output file common prefix> <Sample ID> CodingExons.vcf.gz

571 2. <Output file common prefix $>$ <Sample ID> CodingExons.vcf.gz.tbi

572 3. <Output file common prefix $><$ Sample ID $>$ CodingExons counts.csv

573 4. <Output file common prefix $\rangle_{-}<$Sample ID $\rangle_{-}$CodingExons_counts.json

574 5. <Output file common prefix $\rangle_{-}<$Sample ID>_CodingExons_summary.csv

575 6. <Output file common prefix $><$ Sample ID ${ }^{-}$CodingExons extended.csv

576 7. <Output file common prefix ${ }^{-}<$Sample ID>_CodingExons_metrics.json

577 8. <Output file common prefix>_<Sample ID>_CodingExons_Consoleoutput.txt

578 9. <Output file common prefix $><$ Sample

579 ID> CodingExons indelSizeDistríbution.txt

$58010 .{ }^{-}$<Output file common prefix> <Sample

581

582

583

584

585

586

587

588

589

590

591

592

593

594

595

596

597

598

599

600

601

ID>_CodingExons_indelsizeDistrib̄utiononPlot.pdf

There is a final performance assay report generated in the form of a tab delimited file as below:

Final_benchmarking_metrics_<current_date>.txt

Another set of 10 files as seen above corresponding to the whole exome regions are generated.

The benchmarking framework generates the following intermediate files:

1. <Output file common prefix>_<Sample ID>_CodingExons_SNPs_TPonly.vCf.gz

2. <Output file common prefix>_<Sample ID>_CodingExons_SNPs_FPonly.vCf.gz

3. <Output file common prefix>_<Sample ID>_CodingExons_SNPs_FNonly.VCf.gz

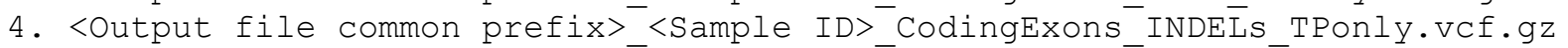

5. <Output file common prefix>_<ample ID>_CodingExons_INDELs_EPonly.VCf.gz

6. <Output file common prefix>_<Sample ID>_CodingExons_INDELs_FNonly.vCf.gz

7. <Output file common prefix>_<SampleID>_CodingExons_índelDistribution.txt

Another set of seven files as seen above corresponding to the whole exome regions are generated.

602

603

604

605 Table S1. Benchmarking metrics for indels of different size ranges in NA12878 (truth set

606 NIST v3.3, total bases $=13728555$ ) for the regions within $\sim 7000$ clinically relevant genes (as

607

608

609 specified in Methods).

\begin{tabular}{|l|l|l|l|l|l|l|l|l|}
\hline $\begin{array}{l}\text { Size of indels } \\
\text { in NA12878 }\end{array}$ & $\begin{array}{l}\text { Truth } \\
\text { total }\end{array}$ & TP & FP & FN & TN & NPA & Precision & Recall \\
\hline $1-10$ & 145 & 139 & 10 & 6 & 13728400 & 100 & 93.29 & 95.86 \\
\hline $11-20$ & 7 & 7 & 0 & 0 & 13728548 & 100 & 100 & 100 \\
\hline $21-50$ & 5 & 5 & 0 & 0 & 13728550 & 100 & 100 & 100 \\
\hline All Indels & 156 & 150 & 10 & 6 & 13728389 & 100 & 93.75 & 96.15 \\
\hline
\end{tabular}

610

611 Table S2. Benchmarking metrics for indels of different size ranges in NA24143 (truth set

612 NIST v3.3, total bases $=12549224$ ) for the regions within $\sim 7000$ clinically relevant genes (as 613 specified in Methods).

614

\begin{tabular}{|l|l|l|l|l|l|l|l|l|}
\hline $\begin{array}{l}\text { Size of indels } \\
\text { in NA24143 }\end{array}$ & $\begin{array}{l}\text { Truth } \\
\text { total }\end{array}$ & TP & FP & FN & TN & NPA & Precision & Recall \\
\hline $1-10$ & 153 & 143 & 16 & 10 & 12549055 & 100 & 89.94 & 93.46 \\
\hline
\end{tabular}


615

616

617

618

619

620

621

622

623

624

625

626

627

628

629

630

631

632

633

634

635

636

637

638

639

\begin{tabular}{|l|l|l|l|l|l|l|l|l|}
\hline $11-20$ & 8 & 8 & 0 & 0 & 12549216 & 100 & 100 & 100 \\
\hline $21-50$ & 3 & 3 & 0 & 0 & 12549221 & 100 & 100 & 100 \\
\hline All Indels & 163 & 153 & 16 & 10 & 12549045 & 100 & 90.53 & 93.87 \\
\hline
\end{tabular}

Table S3. Benchmarking metrics for indels of different size ranges in NA24149 (truth set NIST v3.3, total bases $=12538042$ ) for the regions within $\sim 7000$ clinically relevant genes (as specified in Methods).

\begin{tabular}{|l|l|l|l|l|l|l|l|l|}
\hline $\begin{array}{l}\text { Size of indels } \\
\text { in NA24149 }\end{array}$ & $\begin{array}{l}\text { Truth } \\
\text { total }\end{array}$ & TP & FP & FN & TN & NPA & Precision & Recall \\
\hline $1-10$ & 156 & 153 & 8 & 3 & 12537878 & 100 & 95.03 & 98.08 \\
\hline $11-20$ & 8 & 8 & 1 & 0 & 12538033 & 100 & 88.89 & 100 \\
\hline $21-50$ & 1 & 1 & 0 & 0 & 12538041 & 100 & 100 & 100 \\
\hline All Indels & 163 & 161 & 9 & 3 & 12537869 & 100 & 94.71 & 98.16 \\
\hline
\end{tabular}

Table S4. Benchmarking metrics for indels of different size ranges in NA24631 (truth set NIST v3.3, total bases $=12808688$ ) for the regions within $\sim 7000$ clinically relevant genes (as specified in Methods).

\begin{tabular}{|l|l|l|l|l|l|l|l|l|}
\hline $\begin{array}{l}\text { Size of indels } \\
\text { in NA24631 }\end{array}$ & $\begin{array}{l}\text { Truth } \\
\text { total }\end{array}$ & TP & FP & FN & TN & NPA & Precision & Recall \\
\hline $1-10$ & 153 & 146 & 16 & 7 & 12808519 & 100 & 90.12 & 95.42 \\
\hline $11-20$ & 5 & 5 & 0 & 0 & 12808683 & 100 & 100 & 100 \\
\hline $21-50$ & 5 & 4 & 0 & 1 & 12808683 & 100 & 100 & 80 \\
\hline All Indels & 162 & 154 & 16 & 8 & 12808510 & 100 & 90.59 & 95.06 \\
\hline
\end{tabular}

Table S5. Benchmarking metrics on the number of indels of different size ranges in NA12878 (truth set NIST v3.3, total bases $=71152019$ ) for the whole exome region s including noncoding exons, splice sites (+/- $20 \mathrm{bp}$ ) and clinically relevant deep intronic regions.

\begin{tabular}{|l|l|l|l|l|l|l|l|l|}
\hline $\begin{array}{l}\text { Size of indels } \\
\text { in NA12878 }\end{array}$ & $\begin{array}{l}\text { Truth } \\
\text { total }\end{array}$ & TP & FP & FN & TN & NPA & Precision & Recall \\
\hline $1-10$ & 5108 & 4704 & 781 & 404 & 71146130 & 100 & 85.76 & 92.09 \\
\hline $11-20$ & 209 & 194 & 13 & 15 & 71151797 & 100 & 93.72 & 92.82 \\
\hline $21-50$ & 52 & 47 & 5 & 5 & 71151962 & 100 & 90.38 & 90.38 \\
\hline All Indels & 5318 & 4910 & 800 & 424 & 71145885 & 100 & 85.99 & 92.03 \\
\hline
\end{tabular}

Table S6. Benchmarking metrics on the number of indels of different size ranges in NA24143 (truth set NIST v3.3, total bases $=65657646$ ) for the whole exome regions including noncoding exons, splice sites (+/- 20 bp) and clinically relevant deep intronic regions.

\begin{tabular}{|l|l|l|l|l|l|l|l|l|}
\hline $\begin{array}{l}\text { Size of indels } \\
\text { in NA24143 }\end{array}$ & $\begin{array}{l}\text { Truth } \\
\text { total }\end{array}$ & TP & FP & FN & TN & NPA & Precision & Recall \\
\hline $1-10$ & 5168 & 4676 & 681 & 492 & 65651797 & 100 & 87.29 & 90.48 \\
\hline $11-20$ & 206 & 184 & 13 & 22 & 65657427 & 100 & 93.40 & 89.32 \\
\hline $21-50$ & 84 & 72 & 5 & 12 & 65657557 & 100 & 93.51 & 85.71 \\
\hline All Indels & 5388 & 4878 & 700 & 526 & 65651542 & 100 & 87.45 & 90.24 \\
\hline
\end{tabular}


645

646

647

648

649

650

651

652

653

654

655

656

657

658

659

660

661

662

663

664

665

Table S7. Benchmarking metrics on the number of indels of different size ranges in NA24149 (truth set NIST v3.3, total bases $=65597266$ ) for the whole exome regions including noncoding exons, splice sites (+/- $20 \mathrm{bp}$ ) and clinically relevant deep intronic regions.

\begin{tabular}{|l|l|l|l|l|l|l|l|l|}
\hline $\begin{array}{l}\text { Size of indels } \\
\text { in NA24149 }\end{array}$ & $\begin{array}{l}\text { Truth } \\
\text { total }\end{array}$ & TP & FP & FN & TN & NPA & Precision & Recall \\
\hline $1-10$ & 5096 & 4578 & 628 & 518 & 65591542 & 100 & 87.94 & 89.84 \\
\hline $11-20$ & 188 & 167 & 17 & 21 & 65597061 & 100 & 90.76 & 88.83 \\
\hline $21-50$ & 68 & 62 & 5 & 6 & 65597193 & 100 & 92.54 & 91.18 \\
\hline All Indels & 5290 & 4763 & 651 & 545 & 65591307 & 100 & 87.98 & 89.70 \\
\hline
\end{tabular}

Table S8. Benchmarking metrics on the number of indels of different size ranges in NA24631 (truth set NIST v3.3, total bases $=65657646$ ) for the whole exome regions including noncoding exons, splice sites (+/- 20 bp) and clinically relevant deep intronic regions.

\begin{tabular}{|l|l|l|l|l|l|l|l|l|}
\hline $\begin{array}{l}\text { Size of indels } \\
\text { in NA24631 }\end{array}$ & $\begin{array}{l}\text { Truth } \\
\text { total }\end{array}$ & TP & FP & FN & TN & NPA & Precision & Recall \\
\hline $1-10$ & 5555 & 5089 & 656 & 466 & 66982776 & 100 & 88.58 & 91.61 \\
\hline $11-20$ & 187 & 178 & 6 & 9 & 66988794 & 100 & 96.74 & 95.19 \\
\hline $21-50$ & 82 & 68 & 8 & 14 & 66988897 & 100 & 89.47 & 82.93 \\
\hline All Indels & 5805 & 5316 & 671 & 489 & 66982511 & 100 & 88.79 & 91.58 \\
\hline
\end{tabular}

\section{Supplemental Data files for:}

Benchmarking workflows to assess performance and suitability of germline variant calling pipelines in clinical diagnostic assays

The benchmarking workflow file and relevant scripts (listed below as additional files) and all output files for five GIAB samples per stage are available in our public repository:

vandhanak/stanford-benchmarking-workflows

\section{Supplemental Files}

1. indelSizeDistribution_Detailed.R

2. benchmarking_truth_set.py

3. verify_variants.py 